\title{
High-fructose and high-fat diet-induced insulin resistance enhances atherosclerosis in Watanabe heritable hyperlipidemic rabbits
}

\author{
Bo Ning ${ }^{1 \dagger}$, Xiaoyan Wang ${ }^{1,2 \dagger}$, Ying Yu ${ }^{3}$, Ahmed Bilal Waqar ${ }^{1}$, Qi Yu ${ }^{4}$, Tomonari Koike ${ }^{3}$, Masashi Shiomi ${ }^{3}$, Enqi Liu ${ }^{5}$,
} Yifei Wang ${ }^{2}$ and Jianglin Fan ${ }^{1,4^{*}}$

\begin{abstract}
Background: Individuals with insulin resistance and resulting impaired glucose tolerance along with type 2 diabetes showed an increased prevalence of atherosclerosis. Our aim in this study was to address whether diet-induced insulin resistance plays any roles in the development of aortic and coronary atherosclerosis in hyperlipidemic rabbits.

Methods: We fed Watanabe heritable hyperlipidemic (WHHL) rabbits with a high-fructose and high-fat diet (HFFD) with restricted normal calories and compared the lesions of both aortic and coronary atherosclerosis with those of control WHHL rabbits fed a normal chow diet.

Results: HFFD-fed WHHL rabbits showed insulin resistance and impaired glucose tolerance accompanied by elevated plasma lipid levels and accumulation of adipose tissue even though their body weight was unchanged compared to the control rabbits. At 8 weeks, the aortic gross lesion area of HFFD-fed WHHL rabbits was increased by $40 \%$ over the controls and their lesions were characterized by increased number of macrophages and smooth muscle cells. At 16 weeks, the lesions of HFFD-fed WHHL rabbits showed more advanced lesions such as lipid core formation and calcification. In addition, coronary atherosclerosis was significantly increased in HFFD-fed WHHL rabbits.
\end{abstract}

Conclusions: These results suggest that insulin resistance accelerates lesion formation of atherosclerosis.

\section{Introduction}

Insulin resistance (IR) is frequently associated with many metabolic diseases such as obesity, hypertriglyceridemia, type 2 diabetes, and metabolic syndrome [1]. Individuals with underlying IR along with impaired glucose tolerance also have an increased prevalence of atherosclerosis and coronary heart disease [2, 3]. However, whether IR is directly involved in the development of atherosclerosis has not yet been fully understood [4, 5]. IR is simply defined as impaired response to insulin of the liver, adipose tissue and skeleton muscles due to impairments of the insulin signaling pathway [3]. In experimental animals, IR can be induced by a diet containing high-fructose and/or high-fat. Such diet feeding can result in IR in

\footnotetext{
* Correspondence: jianglin@yamanashi.ac.jp

${ }^{\dagger}$ Equal contributors

'Department of Molecular Pathology, Interdisciplinary Graduate School of Medicine and Engineering, University of Yamanashi, Yamanashi, Japan

${ }^{4}$ Department of Pathology, Xi'an Medical University, Xi'an, China

Full list of author information is available at the end of the article
}

rats $[6,7]$ but not in low-density lipoprotein (LDL) receptor-deficient mice [8]. Although rats are useful for the study of IR, hyperinsulinemia, and hyperglycemia, they are resistant to diet-induced hypercholesterolemia and atherosclerosis because their lipoprotein metabolism is largely different from humans. It is well known that unlike humans, rats as well as mice, are deficient in cholesteryl ester transfer protein (CETP), an important regulator of lipoprotein metabolism and their major lipoproteins are high-density lipoproteins (HDLs) rather than LDLs. Therefore, it is difficult to use these animal models to elucidate the relationship between IR and atherosclerosis.

To examine whether IR plays any roles on atherosclerosis, we performed the current study using Watanabe heritable hyperlipidemic (WHHL) rabbits. Like humans but unlike rodents (rats and mice), rabbits have abundant plasma CETP and their lipoprotein profiles are LDL-rich [9]. WHHL rabbits are genetically deficient in low-density-lipoprotein (LDL) receptor due to a spontaneous 4-amino-acid deletion in the cysteine-rich 
ligand-binding domain in exon 4 of the LDL receptor and develop hyperlipidemia and spontaneous atherosclerosis on a normal chow diet $[10,11]$. WHHL rabbits have been extensively used for the study of hypercholesterolemia and atherosclerosis [12, 13]. In the current study, we fed WHHL rabbits with a diet containing high fructose and fat (HFFD) in attempt to elucidate the roles of IR on early-stage atherosclerosis and advanced atherosclerosis. Our results showed that even when consuming normal numbers of calories similar to the normal chow diet, an HFFD induces IR and enhances the development of aortic and coronary atherosclerosis in WHHL rabbits.

\section{Methods}

\section{Animals and diets}

Watanabe heritable hyperlipidemic (WHHL) MI rabbits [14] (male, 3 months old) provided by Kobe University, Japan, were randomly divided into two groups. The control group $(\mathrm{n}=15)$ was fed a standard chow diet (CR-3, CLEA Japan), which contains $17.3 \%$ protein, $3.9 \%$ vegetable fat, $13.6 \%$ fiber, $8.7 \%$ ash, $48.9 \%$ nitrogen-free extract, and $7.6 \%$ moisture by weight, and its physiological fuel value is approximately $2.997 \mathrm{kcal} / \mathrm{g}$. Control WHHL rabbits $(2.0 \sim 2.5 \mathrm{~kg} \mathrm{BW})$ consumed about $70 \sim$ 90 g chow diet daily, equivalent to average $210 \sim 270$ $\mathrm{kcal} /$ day/animal. The high-fructose and high-fat diet (HFFD) group $(\mathrm{n}=14)$ was fed a chow diet supplemented with $30 \%$ fructose and $10 \%$ coconut oil (91\% saturated fatty acids by weight) because HFFD diet can induce more prominent insulin resistance than a diet containing fructose alone. The HFFD was prepared by Oriental Yeast Com. Tokyo. Compared to a standard chow diet, HFFD was rich in sugar and fat but with relatively less protein and fibers. To avoid the excess gain of body weight induced by HFFD, which may complicate the experiments, we fed HFFD group with restricted diets containing approximately similar calories as the control group for 8 weeks (designated as a "short term" to evaluate the early-stage atherosclerosis) and 16 weeks (designated as a "long term" to evaluate advanced lesions of atherosclerosis). The body weight and daily food consumption were monitored during the experiment. All rabbits were housed in individual cages in a room with controlled temperature $\left(22-24{ }^{\circ} \mathrm{C}\right)$ on a $12 \mathrm{~h}$ light/dark cycle. This study was approved by the Animal Use and Care Committee of the University of Yamanashi and also conformed to the Guide for the Care and Use of Laboratory Animals published by the US National Institutes of Health.

\section{Analysis of plasma lipid and glucose metabolism}

During the experiment, plasma total cholesterol (TC), triglycerides (TG) and high-density-lipoprotein cholesterol
(HDL-C) were determined weekly using Wako assay kits (Wako Pure Chemical Industries, Osaka, Japan) [15]. For the analysis of lipoprotein profiles, plasma lipoproteins were isolated by ultracentrifugation and resolved by electrophoresis in $1 \%$ agarose universal gels (Helena Laboratories, Saitama, Japan) [16]. Plasma lipoproteins were also analyzed using high-performance liquid chromatography (HPLC) by Skylight Biotech, Inc. (Tokyo, Japan) [17]. We measured the rate of VLDL secretion in fasting animals in vivo using Triton WR-1339 [18]. Plasma levels of lipid peroxidation products were measured using a TBARS assay kit (Cayman Chemical Co., Ann Arbor, MI).

To examine the deleterious effects of HFFD on glucose metabolism and insulin response, we performed intravenous glucose tolerance test (IVGTT) and intravenous insulin tolerance test (IVITT) at 4,8 and 16 weeks using a method reported previously [19]. The rabbits were injected with glucose solution ( $0.6 \mathrm{~g} / \mathrm{kg}$ body weight) intravenously after $16 \mathrm{~h}$ of fasting, and then blood samples were drawn at $5,10,15,20,30,45,60,75$ and $120 \mathrm{~min}$. For IITT, after the intravenous injection of insulin $(1 \mathrm{U} / \mathrm{kg}$ body weight, Novo Nordisk Pharmaceutical Co., Tokyo, Japan), blood samples were collected at 15, 30, 45, 60, 90 and $120 \mathrm{~min}$. The glucose and insulin concentrations were measured using Wako glucose assay kits and rabbit insulin ELISA kits (Shibayagi Co., Ltd., Gunma, Japan).

\section{Pathological analysis}

Rabbits were euthanized by an overdose injection of sodium pentobarbital $(100 \mathrm{mg} / \mathrm{kg})$ and the adipose tissue (both subcutaneous and visceral adipose) was collected and weighed wet. For histological analysis, adipose

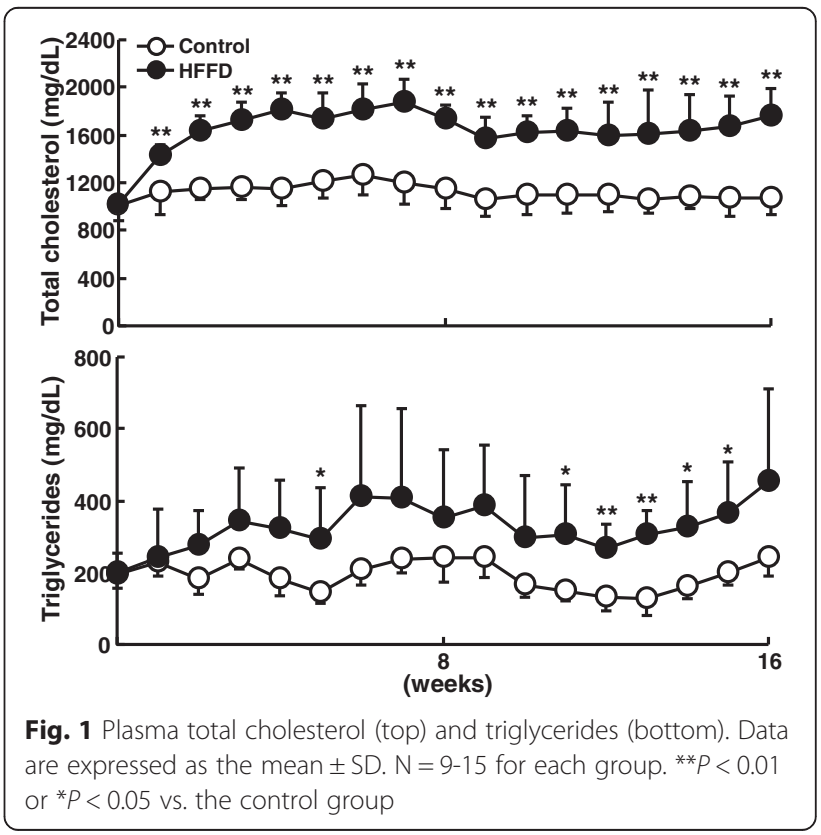


tissue, along with liver and pancreas, was fixed in $10 \%$ buffered formalin. After paraffin-embedding, sections (3 $\mu \mathrm{m}$ thick) were routinely cut and stained with hematoxylin and eosin (H\&E). Adipocyte size was evaluated under a light microscope using H\&E-stained sections, as described previously [19]. Briefly, more than 300 adipocytes from each section were randomly measured for cellular diameter by two observers blindly, using an image analysis system (Winroof ver 6.4, Mitani Co., Tokyo) [19]. The average diameter of adipocytes in each group was calculated and the cell size distribution was analyzed and expressed as a percentage. Islet number and islet area on the sections immunohistochemically stained with insulin monoclonal antibody were calculated as described previously [20].

\section{Analysis of aortic and coronary atherosclerosis}

The aorta and heart were isolated from each rabbit. Aortic trees were opened and fixed in $10 \%$ buffered formalin. After formalin fixation, the whole aortas were stained with Sudan IV and photographed for evaluation of the gross size of atherosclerotic lesions [14]. The surface sudanophilic area of each aorta segment was measured using an image analysis system. For histological analysis, serial sections $(3 \mu \mathrm{m}$ thick) were stained with $\mathrm{H} \& \mathrm{E}$, Elastica van Gieson (EVG) and immunohistochemically stained with
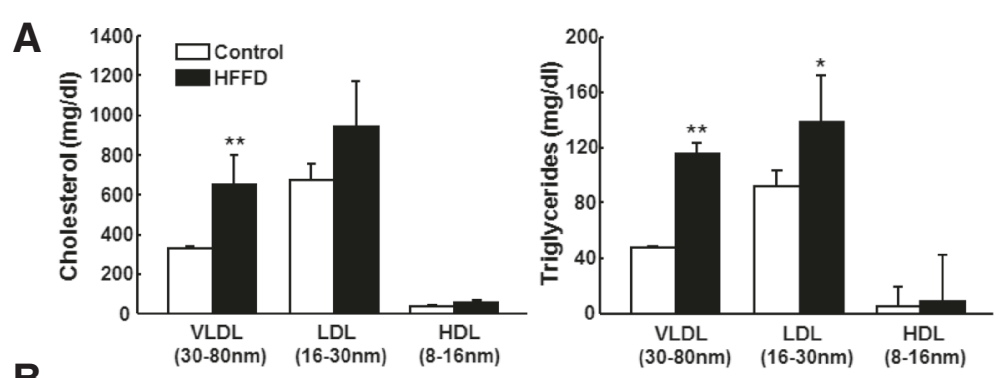

B
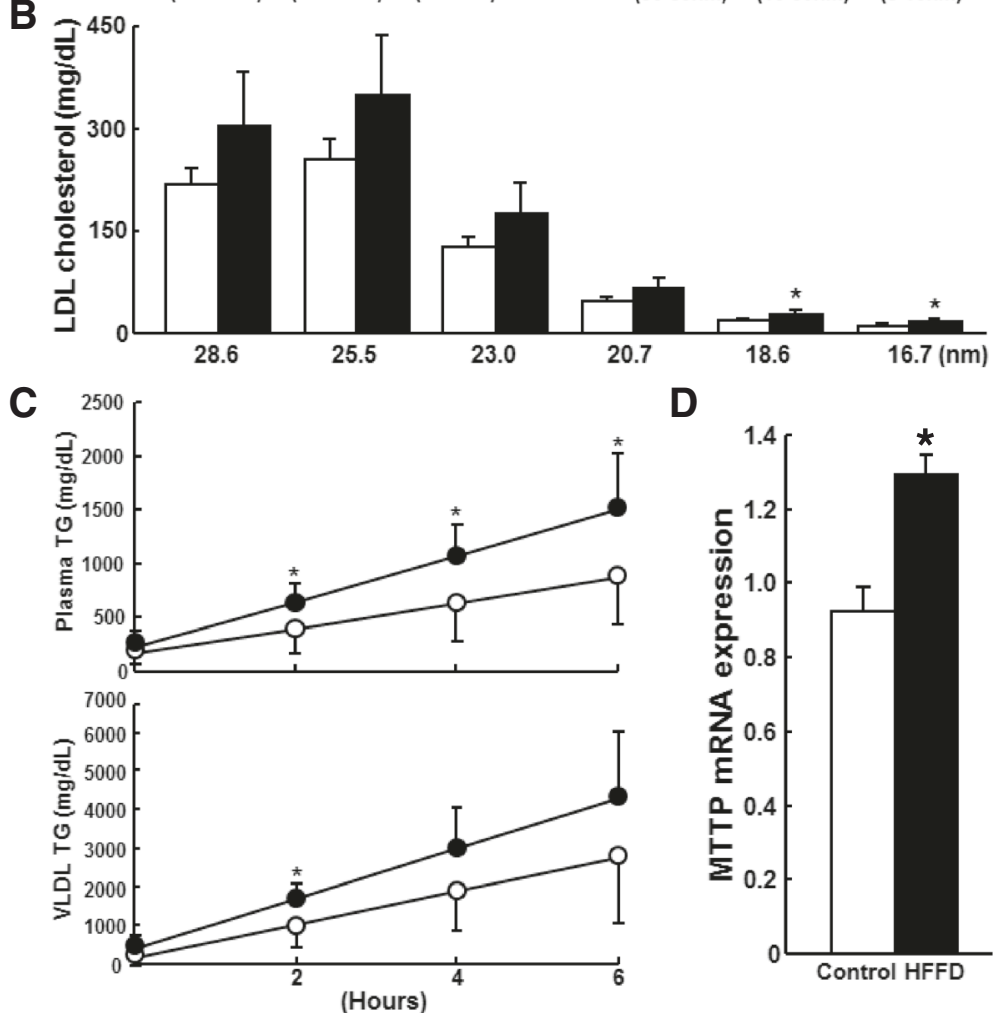

D

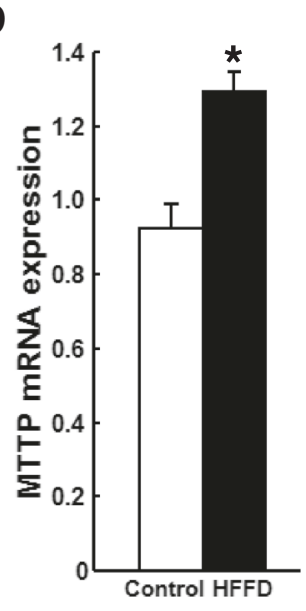

Fig. 2 a-b Quantitative analysis of VLDL, LDL and HDL contents (a) and different-sized LDL fractions (b). Plasma lipoproteins (at 8 weeks) were fractionated according to their size by HPLC and lipid contents of each fraction were quantified as described in the Methods. Data are expressed as the mean $\pm S D$. $n=5$ for each group. ${ }^{*} P<0.01$ or ${ }^{*} P<0.05$ vs. the control group. $\mathbf{c}$ - $\mathbf{d}$ Analysis of hepatic VLDL synthesis and MTTP expression. Post-Triton VLDL production rate in fasting rabbits at 8 weeks after HFFD or chow diet feeding was measured. Blood was drawn at 0 minutes (before administration of Triton WR-1339) and 2, 4 and 6 hours after Triton WR-1339 injection. Triglyceride contents of total plasma (C, top) and VLDLs $(\mathrm{d}<1.006 \mathrm{~g} / \mathrm{mL})(\mathrm{C}$, bottom) were quantified. Hepatic mRNA expression of microsomal triglyceride transfer protein (MTTP) was analyzed using real-time RT-PCR (D). Data are expressed as the mean \pm SD. $n=5$ for each group. ${ }^{*} P<0.05$ vs. the control group 
RAM11 (working dilution 400X) and HHF35 (200X) monoclonal antibodies against macrophages and smooth muscle $\alpha$-actin (Dako) [14]. The microscopic lesion area, macrophages and SMCs in the lesions were quantified and early-stage lesions and advanced lesions were classified and analyzed using an image analysis system [14]. Advanced lesions refer to those which to contain a typical fibrotic cap and a lipid or necrotic core with or without calcification [14]. Rabbit hearts were sectioned and coronary atherosclerosis was analyzed and expressed as stenosis $\%$, as described previously [15].

\section{Real-time reverse transcriptase-polymerase chain reaction} (RT-PCR)

Total RNA from the liver was isolated using Trizol reagent (Invitrogen, Life Technologies, Inc., Carlsbad, CA) and then mRNA expression of nuclear factor-like 2 (Nrf2), superoxide dismutase 1 (SOD1) and microsomal triglyceride transfer protein (MTTP) was analyzed by real-time reverse transcriptase (RT)-polymerase chain reaction (PCR) [14]. The panel of primers used for analyzing the gene expression is shown in Additional file 1: Table S1.

\section{Statistical analysis}

Statistical analyses were performed using SPSS 12.0 software. All data are expressed as the mean \pm SD. Statistical significance data was determined for parametric data by Student's $t$-test and for non-parametric data by Mann-Whitney $U$-test. In all cases, a $p$ value of less than 0.05 was considered statistically significant.

\section{Results}

\section{Effect of HFFD on plasma lipids and lipoproteins}

There was no change in body weight between the two groups $(2.28 \pm 0.32$ in the control vs $2.32 \pm 0.21$ $\mathrm{kg}$ in HFFD at $0 \mathrm{w} ; 2.40 \pm 0.28$ vs $2.46 \pm 0.24 \mathrm{~kg}$ at $8 \mathrm{w}$, and $2.56 \pm 0.15$ vs $2.62 \pm 0.14 \mathrm{~kg}$ at $16 \mathrm{w}$ ) as they consumed similar numbers of calories of each diet (Additional file 1: Figure S1). It should be noted that this HFFD contained relative shortage of other components (such as proteins and fibers) compared to the standard chow diet. In the current study; we fed both groups with restricted amount of each diet containing equal numbers of calories to minimize the HFFD effect on the body weight.

Moreover, HFFD-fed rabbits had higher plasma TC and TG levels (Fig. 1) than control rabbits throughout the experimental period, but their HDL-C levels were unchanged (data not shown). Analysis of lipoprotein profiles by HPLC and ultracentrifugation revealed that increased plasma TC and TG levels in the HFFD group were attributable to increased apolipoprotein (apo)-B containing particles, very-low-density lipoproteins (VLDLs) and LDLs because both TC and TG contents in these fractions were significantly increased compared with those of control rabbits (Fig. 2a and Additional file 1: Figure S2). Quantitative analysis of LDL particles showed that the levels of all LDL particles were increased in the HFFD group, especially those of small-sized LDL particles (Fig. 2b). To illustrate the possible mechanisms responsible for increased plasma VLDLs and LDLs induced by HFFD, we measured the rate of hepatic VLDL secretion in vivo using Triton WR-1339 to block the hydrolysis of TG-rich lipoproteins by lipoprotein lipase [18]. As shown in Fig. 2c-d, HFFD increased the VLDL synthesis rate, which was accompanied by higher expression of MTTP mRNA, the rate-limiting mediator for VLDL production in the liver.

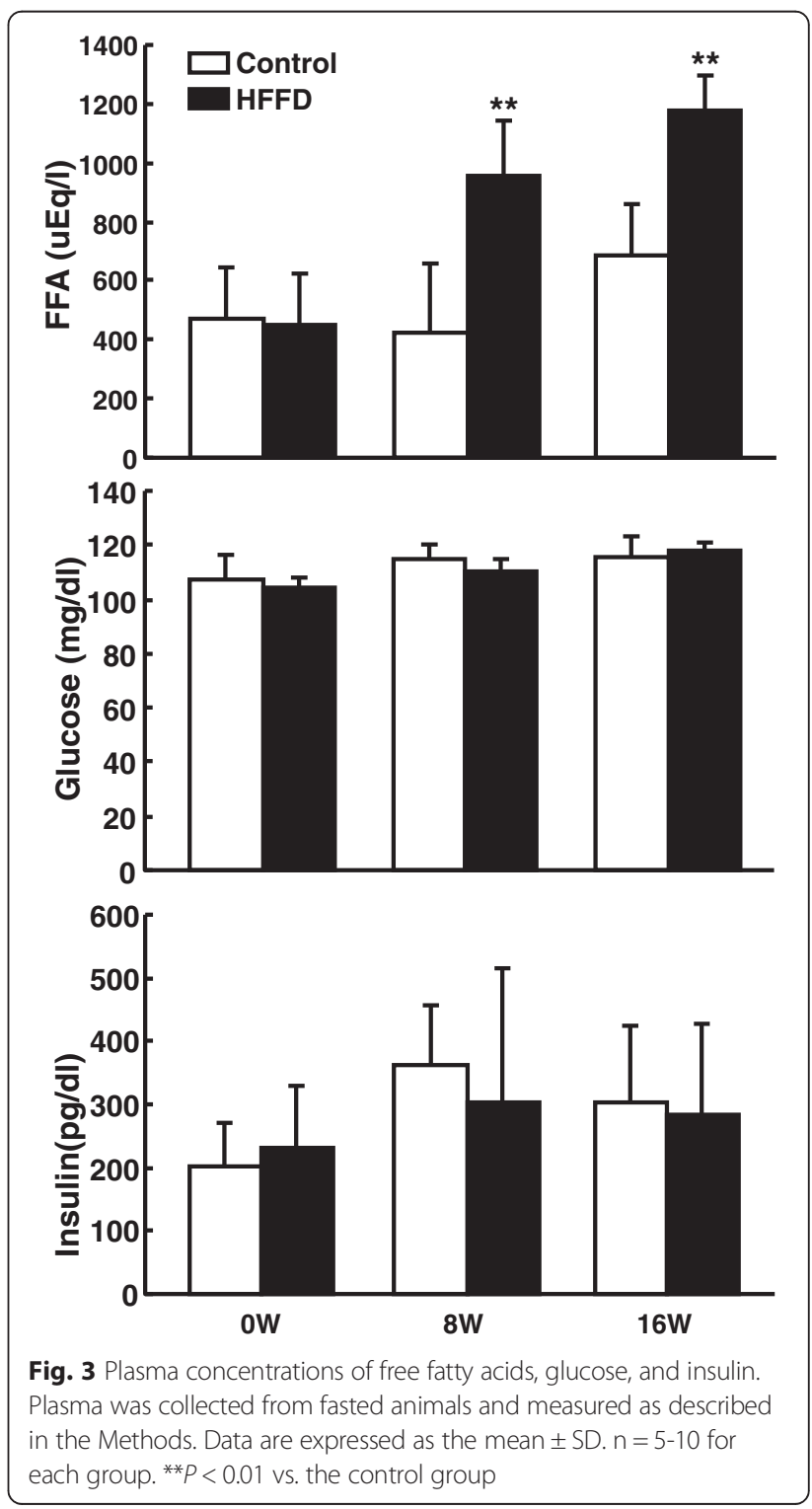




\section{Effect of HFFD on glucose and insulin metabolism}

HFFD feeding increased the plasma levels of free fatty acids but did not change the plasma glucose and insulin levels compared with those in the control (Fig. 3). In addition, HFFD led to impaired glucose and insulin response. As shown in Fig. 4, HFFD-fed rabbits exhibited delayed capacity for clearing glucose from the circulation when challenged with a glucose bolus, which was accompanied by higher insulin secretion and reduced insulin response. This reduced response to insulin induced by HFFD was first noted at 4 weeks and continued at 8 and 16 weeks (Additional file 1: Figure S3). However, the glucose intolerance did not get worse over the course (4, 6,8 weeks), suggesting that aging may influence glucose tolerance in WHHL rabbits.

Then, we examined the histological changes of the pancreas and found that islet number and area of the pancreas of the HFFD group were significantly increased by $58 \%$ and $65 \%$ compared with those of the control at 8 weeks (Additional file 1: Figure S4). Taken together, these results showed that the HFFD group exhibited insulin resistance.

\section{Pathological examination of liver and adipose tissue}

Pathological examinations revealed that the liver of HFFD-fed rabbits showed hepatic steatosis while the liver weight was unchanged (Additional file 1: Figure S5). At 8 weeks, 7 of 9 HFFD-fed rabbits (77\%) showed remarkable fatty liver, in contrast to control rabbits, in which only 2 of 10 rabbits (20\%) had mild and focal hepatic steatosis (Additional file 1: Figure S5), and similar results were obtained at 16 weeks (data not shown). We next examined oxidative stress-associated gene expression in the liver using real-time RT-PCR and found that Nrf2 mRNA expression was increased whereas SOD1 was reduced in HFFD-fed rabbits compared to the control (Additional file 1: Figure S5). In addition, plasma levels of TBARS were significantly increased in HFFD-fed group (Additional file 1: Figure S5).

We next examined adipose tissue and found that the amount of adipose accumulation of the HFFD group was significantly increased in both subcutaneous (1.8- and 1.28 -fold increases over the control at 8 and 16 weeks) and visceral regions (2.37- and 1.21-fold increases over the control at 8 and 16 weeks) (Fig. 5a-b), although their body weights were similar (Additional file 1: Figure S1). Histological and morphometric analysis showed that adipose tissue of the HFFD group was characterized by an increased number of large-sized adipocytes, which leads to the cell size distribution being shifted towards a larger size in both visceral and subcutaneous regions (Fig. 5c-d). We made a thorough evaluation of adipose tissue histology and did not find prominent increase of macrophage infiltration in the HFFD group compared to the control group. This may be not surprising because HFFD feeding did not induce remarkable obesity in rabbits.

We also measured blood pressure and heart rate [20], but did not find any differences between the two groups (Additional file 1: Figure S6).

\section{Aortic atherosclerosis}

At 8 weeks, en face lesion area was increased by $40 \%$ $(\mathrm{p}<0.0 .5)$ in the whole aorta of the HFFD group compared with that in the controls (Fig. 6a), with $11 \%$ $(\mathrm{p}=0.14), 60 \%(\mathrm{p}=0.052)$, and $70 \%(\mathrm{p}=0.08)$ increase
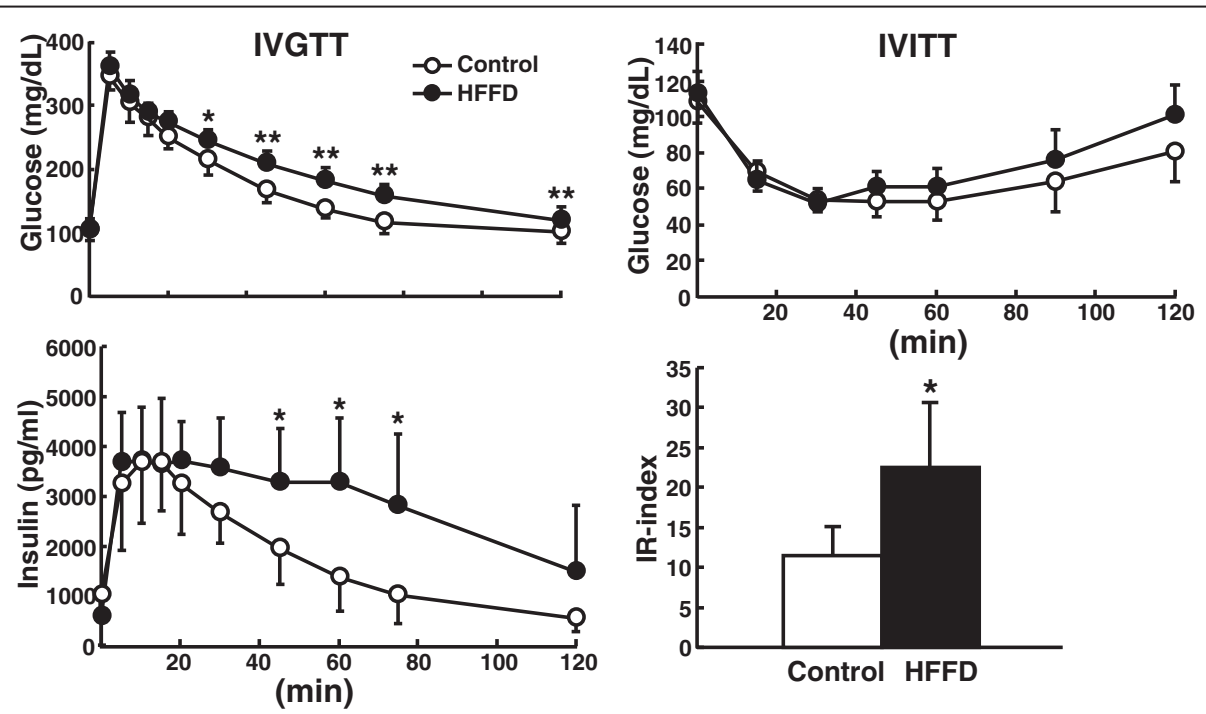

Fig. 4 Evaluation of glucose and insulin metabolism by IVGTT and IVITT. Rabbits at 4 weeks after HFFD feeding were intravenously injected with either glucose (left) or insulin (right) as described in the Methods. The changes in plasma glucose levels (top left and right) and insulin levels (bottom left) were determined. Insulin resistance index (IR-index) was calculated [19]. Data are expressed as the mean \pm SD. $n=6$ for each group. ** $P<0.01$ or ${ }^{*} P<0.05$ vs. the control group 


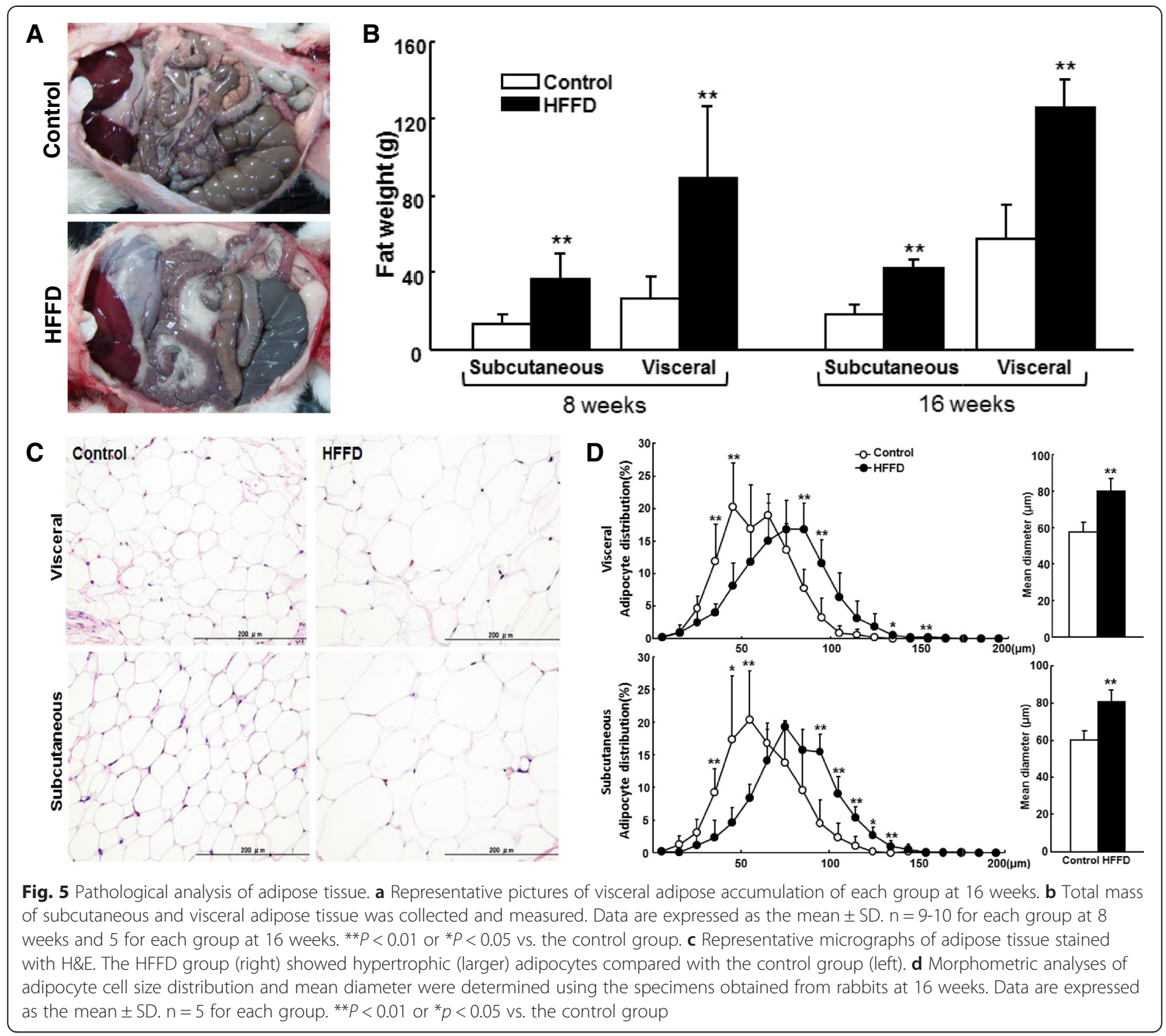

in aortic arch, thoracic and abdominal aorta, but the differences were not statistically significant. Histological examination along with immunohistochemical staining showed that the atherosclerotic lesions of both HFFD and control groups were mainly composed of fatty streaks (Fig. 6b); however, the microscopic lesion size of the total aorta was increased in the HFFD group due to increased numbers of both macrophages (55\% increase over the control) and smooth muscle cells (54 $\%$ increase over the control), even though this was not statistically significant $(\mathrm{p}=0.05)$ (Fig. 6c).

At 16 weeks, the aortic surfaces of both groups were almost completely covered by the atherosclerotic lesions, while only the lesion area of the aortic arch of HFFD group was greater than that of the control group $(\mathrm{p}<0.01)$ (Fig. 7a). In contrast to the lesions at 8 weeks, in which early-stage lesions are predominant as described above, the lesions at 16 weeks of both groups became more advanced and many parts of the aortas showed necrotic or lipid core formation and calcification in addition to fatty streaks (Fig. 7b). Similarly to lesions at 8 weeks, HFFD feeding for 16 weeks increased microscopic lesion, with tendencies for increasing macrophages, and smooth muscle cells although these differences were not statistically significant (Fig. 7c). Nevertheless, the advanced lesions and the lipid core size of HFFD-fed WHHL rabbits were significantly increased compared to the control group (Fig. 7c).

\section{Coronary atherosclerosis}

In addition to the aorta, the HFFD led to a significant increase of coronary atherosclerosis. There was a $60 \%$ increase of coronary stenosis (both left and right coronary arteries) at 8 weeks and a $103 \%$ increase at 16 weeks compared with that of the controls (Fig. 8). 


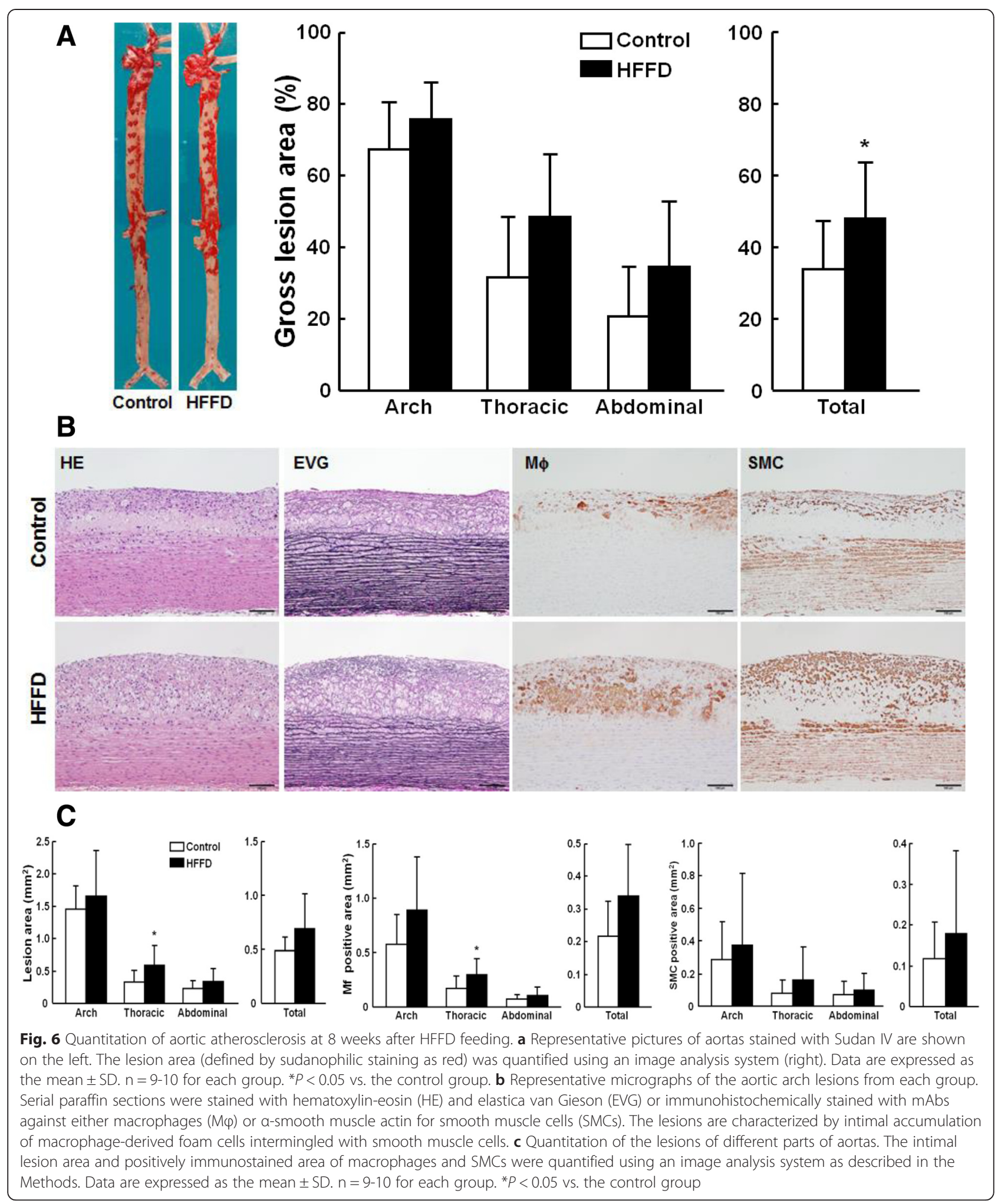

\section{Discussion}

In the current study, we fed WHHL rabbits with two kinds of diets: HFFD (rich in sugar and fat with reduced protein and fibers) and standard chow diet (protein- and fiber-rich). Although both groups consumed an equal amount of calorie of each diet, HFFD feeding led to prominent IR accompanied by elevated plasma lipids, hepatic steatosis and adipose accumulation, even though 

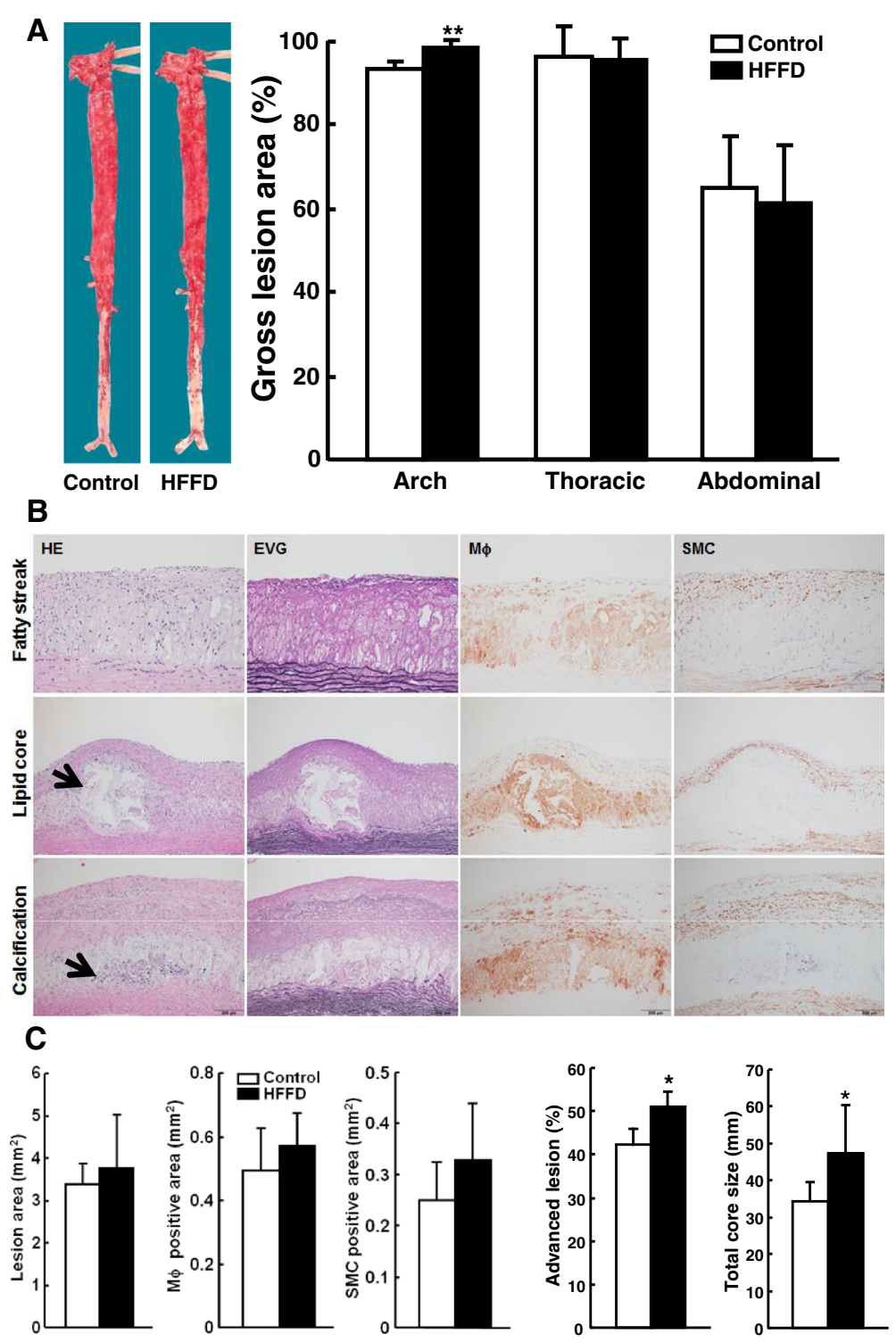

Fig. 7 Quantitation of aortic atherosclerosis at 16 weeks after HFFD feeding. a Representative pictures of aortas stained by Sudan IV are shown on the left. The lesion area (defined by sudanophilic staining as red) was quantified using an image analysis system (right). Data are expressed as the mean \pm SD. $n=5$ for each group. ${ }^{* *} P<0.01$ vs. the control group. b Representative micrographs of the aortic arch lesions from each group. Serial paraffin sections were stained with hematoxylin-eosin (HE) and elastica van Gieson (EVG) or immunohistochemically stained with mAbs against either macrophages $(M \varphi)$ or a-smooth muscle actin for smooth muscle cells (SMCs). Histologically, there are two types of lesion: (1) early-stage lesions (fatty streaks) are mainly composed of foam cells as shown at 8 weeks above (top); and (2) advanced lesions are characterized by the formation of lipid core or necrotic cores covered by a fibrous cap. In addition, calcification is often seen (middle and bottom indicated by arrowheads). c Quantitation of aortic arch lesions, macrophages and smooth muscle cells, the early stage lesions, and advanced lesions. Data are expressed as the mean $\pm S D . n=5$ for each group. ${ }^{*} P<0.05$ vs. the control group

the body weight was unchanged. Increased plasma levels of lipids are basically caused by high uptake of free fatty acids into the liver where they can be synthesized into VLDLs, which are accumulated in the plasma. At the same time, VLDL accumulation was further enhanced due to delayed catabolism of VLDLs in the context of deficiency of LDL receptors in WHHL rabbits. It should be pointed out that high-fat diet feeding did not increase plasma lipids in wild-type rabbits which have normal LDL receptors [20]. This notion is further supported by our Triton experiments along with RT-PCR analysis showing that there was increased production of VLDLs and expression of hepatic MTTP mRNA in HFFD-fed WHHL rabbits. Increased free fatty acids in the 

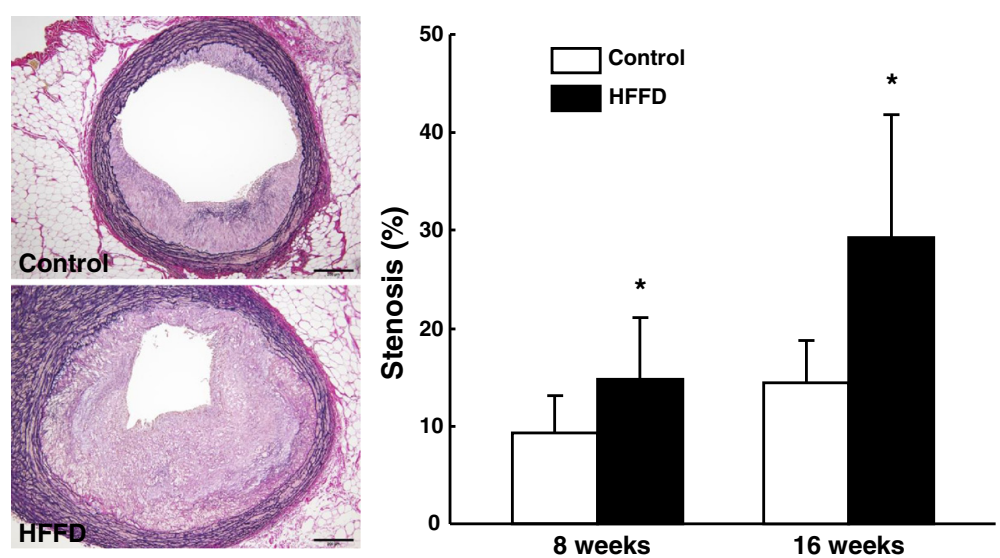

Fig. 8 Quantitation of coronary atherosclerosis. Representative micrographs of the left coronary lesions from each group (EVG staining) are shown on the left and the lesion size (expressed as stenosis \%) of both right and left coronary arteries is shown on the right. Data are expressed as the mean \pm SD. $n=9-10$ for each group at 8 weeks and 5 for each group at 16 weeks. ${ }^{*} P<0.05$ vs. the control group

circulation can also induce fatty liver and adipose accumulation, and reduce the response to insulin thereby causing IR [3]. A high flux of fructose to the liver, the main organ capable of metabolizing this simple carbohydrate, perturbs glucose metabolism and glucose uptake pathways, and leads to a significantly enhanced rate of de novo lipogenesis and TG synthesis, driven by the high flux of glycerol and acyl portions of TG molecules from fructose catabolism. This conjecture is implicated by the finding that HFFD-fed WHHL rabbits had prominent fatty liver compared with the control group. In addition, there was increased Nrf2 expression accompanied by low SOD1 expression in the liver, suggesting that HFFD may induce hepatic insulin resistance and subsequent steatosis through the enhancement of increased free fatty acid influx into the portal system and hepatic oxidative stress $[21,22]$.

Secondly, HFFD-fed WHHL rabbits had greater aortic and coronary atherosclerosis than the control WHHL rabbits. In the current study, we compared the lesions of atherosclerosis after feeding on HFFD for 8 and 16 weeks in an attempt to investigate the shortterm effects of HFFD-induced IR on the early-stage lesions as well as the long-term effects of HFFD-induced IR on the advanced lesions of atherosclerosis. At 8 weeks, aortic atherosclerosis was increased by $40 \%$ in the HFFD group compared with that in the control group and the lesions were characterized by increased macrophages and smooth muscle cells, suggesting that HFFD feeding affects the cellular components in the early-stage lesions. Interestingly, at 16 weeks, aortic surface atherosclerotic lesions became saturated due to the presence of hypercholesterolemia in both groups, so the differences of the total aortic lesion size between HFFD groups and controls are not as prominent as those shown at 8 weeks. In spite of this, the lesions at 16 weeks are characterized by increased advanced lesions, such as lipid cores and calcification. Increased lipid cores and calcification in the lesions of the HFFD group indicate that the lesions become more vulnerable and more susceptible to complications. In the current study, we also demonstrated that HFFD induced the enhancement of coronary stenosis. It should be pointed out that; however, enhanced aortic and coronary atherosclerosis induced by HFFD occurs through several mechanisms, among which IR may play the major roles. For example, IR can be an antecedent or cause of elevated levels of plasma lipids, adipose tissue accumulation and fatty liver as described above. It is also likely that these disorders exhibit a deleterious effect on insulin sensitivity thereby enhancing atherosclerosis. Therefore, it is conceivable that IR together with elevated plasma lipids and adipose accumulation [20, 23, 24] exhibits combinational roles in enhancing the progression of atherosclerosis in HFFD-fed WHHL rabbits. In addition, there is an increase of plasma LDLs, especially small-sized LDLs, in HFFD-fed WHHL rabbits, which is often seen in metabolic syndrome patients. It has been shown that small LDLs are more atherogenic than large ones because they are trapped in the arterial intima more easily and become oxidized [25, 26]. Unlike WHHL rabbits, fructose-fed LDL receptor-deficient mice failed to develop IR while their aortic atherosclerosis was greater than Western-type diet-fed counterpart mice in which IR was present [8]. Apparently, LDL receptor-deficient mice are different from WHHL rabbits in terms of lipid metabolism and susceptibility to atherosclerosis as shown in the current study. 


\section{Conclusion}

We have shown that HFFD-fed WHHL rabbits showed enhancement of the atherosclerosis. The lesions of HFFD-fed WHHL rabbits are rich in macrophages at the early stage and characterized by increased lipid core formation in the advanced lesions. This finding is of clinical importance because our results suggest that even without apparent obesity as shown in the current study, HFFD (even on a normal calorie range) can be still harmful to insulin sensitivity thereby affecting atherosclerosis.

\section{Additional file}

Additional file 1: Table S1. Primers used for Real-Time RT-PCR. Table S2. Average daily food and calorie intake. Figure S1. Food intake, calorie intake and body weight of WHHL rabbits are shown. Figure S2. Analysis of lipoprotien density fractions. Figure S3. IVGTT and IVITT measured at 8 weeks and 16 weeks. Figure S4. Immunohistochemical staining and morphometric analyses of the islet size and area of pacreas. Figure S5. Micrographs of the liver (upper left), liver weight (upper right), hepatic mRNA expression of Nfr2 and SOD1 (lower left and middle) and plasma level of TBARS (bottom right) are shown. Figure S6. Blood pressure and heart rate (beat/min) were measured at 16 weeks using the method described in Ref. 13. (PDF 545 kb)

\section{Abbreviations}

CETP: Cholesteryl ester transfer protein; EVG: Elastica van Gieson; HDL-C: High density lipoprotein-cholesterol; H\&E: Hematoxylin and eosin; HFFD: High fat and fructose diet; HPLC: High-performance liquid chromatography; IR: Insulin resistance; IVGTT: Intravenous glucose tolerance test; IVITT: Intravenous insulin tolerance test; LDL: Low density lipoprotein; MTTP: Microsomal triglyceride transfer protein; Nrf2: Nuclear factor-like 2; RT-PCR: Reverse transcription polymerase chain reaction; SMC: Smooth muscle cell; SOD1: Superoxide dismutase 1; TC: Total cholesterol; TG: Triglycerides; VLDL: Very low density lipoprotein; WHHL rabbits: Watanabe heritable hyperlipidemic rabbits.

\section{Competing interests}

The authors declare that they have no competing interests.

\section{Authors' contributions}

$\mathrm{EL}, \mathrm{YW}$ and JF conceived and designed the experiments. MS provided animals. BN, XW, ABW, TK, YY, and QY performed the experiments. BN, XW, and $A B W$ analyzed the data and performed statistical analysis. YW and JF wrote the paper. JF acquired the funding. All authors read and approved the final manuscript.

\section{Acknowledgements}

We thank Niimi M, Sumii A, Satoh K (University of Yamanashi) for their help throughout the experiments. The work was supported in part by grants-in-aid for scientific research from the Ministry of Education, Culture, Sports and Technology, Japan (22390068, 25670190, and 15H04718 to JF).

\section{Author details}

'Department of Molecular Pathology, Interdisciplinary Graduate School of Medicine and Engineering, University of Yamanashi, Yamanashi, Japan. ${ }^{2}$ Biomedicine Research and Development Center, Jinan University, Guangzhou, China. ${ }^{3}$ Institute for Experimental Animals, Kobe University School of Medicine, Kobe, Japan. ${ }^{4}$ Department of Pathology, Xi'an Medical University, Xi'an, China. ${ }^{5}$ Research Institute of Atherosclerotic Disease and Laboratory Animal Center, Xi'an Jiaotong University School of Medicine, Xi'an, China.
Received: 26 March 2015 Accepted: 29 July 2015

Published online: 12 August 2015

\section{References}

1. Nigro J, Osman N, Dart AM, Little PJ. Insulin resistance and atherosclerosis. Endocr Rev. 2006;27:242-59.

2. Howard G, O'Leary DH, Zaccaro D, Haffner S, Rewers M, Hamman R, et al. Insulin sensitivity and atherosclerosis. The Insulin Resistance Atherosclerosis Study (IRAS) Investigators. Circulation. 1996;93:1809-17.

3. Ginsberg HN. Insulin resistance and cardiovascular disease. J Clin Invest. 2000;106:453-8

4. Kahn R, Buse J, Ferrannini E, Stern M. The metabolic syndrome: time for a critical appraisal: joint statement from the American Diabetes Association and the European Association for the Study of Diabetes. Diabetes Care. 2005;28:2289-304.

5. Semenkovich CF. Insulin resistance and atherosclerosis. J Clin Invest. 2006;116:1813-22.

6. Hwang IS, Ho H, Hoffman BB, Reaven GM. Fructose-induced insulin resistance and hypertension in rats. Hypertension. 1987:10:512-6.

7. Tran LT, Yuen VG, McNeill JH. The fructose-fed rat: a review on the mechanisms of fructose-induced insulin resistance and hypertension. Mol Cell Biochem. 2009:332:145-59.

8. Merat S, Casanada F, Sutphin M, Palinski W, Reaven PD. Western-type diets induce insulin resistance and hyperinsulinemia in LDL receptor-deficient mice but do not increase aortic atherosclerosis compared with normoinsulinemic mice in which similar plasma cholesterol levels are achieved by a fructose-rich diet. Arterioscler Thromb Vasc Biol. 1999;19:1223-30.

9. Fan J, Kitajima S, Watanabe T, Xu J, Zhang J, Liu E, et al. Rabbit models for the study of human atherosclerosis: From pathophysiological mechanisms to translational medicine. Pharmacol Ther. 2015;146C:104-19.

10. Yamamoto T, Bishop RW, Brown MS, Goldstein JL, Russell DW. Deletion in cysteine-rich region of LDL receptor impedes transport to cell surface in WHHL rabbit. Science. 1986;232:1230-7.

11. Watanabe Y. Serial inbreeding of rabbits with hereditary hyperlipidemia (WHHL-rabbit). Atherosclerosis. 1980;36:261-8.

12. Shiomi M, Ito T, Yamada S, Kawashima S, Fan J. Development of an animal model for spontaneous myocardial infarction (WHHLMI rabbits). Arterioscler Thromb Vasc Biol. 2003:23:1239-44

13. Shiomi M, Fan J. Unstable coronary plaques and cardiac events in myocardial infarction-prone Watanabe heritable hyperlipidemic rabbits: questions and quandaries. Curr Opin Lipidol. 2008;19:631-6.

14. Li S, Liang J, Niimi M, Bilal Waqar A, Kang D, Koike T, et al. Probucol suppresses macrophage infiltration and MMP expression in atherosclerotic plaques of WHHL rabbits. J Atheroscler Thromb. 2014;21:648-58.

15. Wang $Y$, Niimi M, Nishijima $K$, Yu Y, Koike T, Kitajima $K$, et al. Human apolipoprotein All protects against diet-induced atherosclerosis in transgenic rabbits. Arterioscler Thromb Vas Biol. 2013;33:224-31.

16. Fan J, Ji Z-S, Huang Y, de Silva H, Sanan D, Mahley R, et al. Increased expression of apolioprotein $\mathrm{E}$ in transgenic rabbits results in reduced levels of very low density lipoproteins and an accumulation of low density lipoproteins in plasma. J Clin Invest. 1998;101:2151-64.

17. Zhang J, Yu Y, Nakamura K, Koike T, Waqar AB, Zhang $X$, et al. Endothelial lipase mediates HDL levels in normal and hyperlipidemic rabbits. J Atheroscler Thromb. 2012;19:213-26

18. Koike T, Kitajima S, Yu Y, Li Y, Nishijima K, Liu E, et al. Expression of human apoAll in transgenic rabbits leads to dyslipidemia: a new model for combined hyperlipidemia. Arterioscler Thromb Vasc Biol. 2009;29:2047-53.

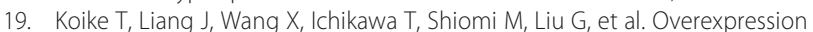
of lipoprotein lipase in transgenic Watanabe heritable hyperlipidemic rabbits improves hyperlipidemia and obesity. J Biol Chem. 2004:279:7521-9.

20. Wagar AB, Koike T, Yu Y, Inoue T, Aoki T, Liu E, et al. High-fat diet without excess calories induces metabolic disorders and enhances atherosclerosis in rabbits. Atherosclerosis. 2010;213:148-55.

21. Bers DM. Cardiac $\mathrm{Na} / \mathrm{Ca}$ exchange function in rabbit, mouse and man: what's the difference? J Mol Cell Cardiol. 2002;34:369-73.

22. Wang D, Chen Y, Chabrashvili T, Aslam S, Borrego Conde LJ, Umans JG, et al. Role of oxidative stress in endothelial dysfunction and enhanced responses to angiotensin $\|$ of afferent arterioles from rabbits infused with angiotensin II. J Am Soc Nephrol. 2003;14:2783-9. 
23. Biddinger SB, Hernandez-Ono A, Rask-Madsen C, Haas JT, Aleman JO, Suzuki R, et al. Hepatic insulin resistance is sufficient to produce dyslipidemia and susceptibility to atherosclerosis. Cell Metab. 2008;7:125-34.

24. Marian AJ. Beta-adrenergic receptors signaling and heart failure in mice, rabbits and humans. J Mol Cell Cardiol. 2006;41:11-3.

25. Witztum JL. The oxidation hypothesis of atherosclerosis. Lancet. 1994;344:793-5.

26. Boren J, Gustafsson M, Skalen K, Flood C, Innerarity TL. Role of extracellular retention of low density lipoproteins in atherosclerosis. Curr Opin Lipidol. 2000;11:451-6.

\section{Submit your next manuscript to BioMed Central} and take full advantage of:

- Convenient online submission

- Thorough peer review

- No space constraints or color figure charges

- Immediate publication on acceptance

- Inclusion in PubMed, CAS, Scopus and Google Scholar

- Research which is freely available for redistribution 